\title{
Spin confinement in the superlattices of graphene ribbons
}

M. Topsakal, H. Sevinçli, and S. Ciraci

Citation: Appl. Phys. Lett. 92, 173118 (2008);

View online: https://doi.org/10.1063/1.2919525

View Table of Contents: http://aip.scitation.org/toc/apl/92/17

Published by the American Institute of Physics

\section{Articles you may be interested in}

A spin-filter device based on armchair graphene nanoribbons

Applied Physics Letters 98, 023106 (2011); 10.1063/1.3537965

Field effect on spin-polarized transport in graphene nanoribbons

Applied Physics Letters 92, 163109 (2008); 10.1063/1.2908207

Bottom-up graphene nanoribbon field-effect transistors

Applied Physics Letters 103, 253114 (2013); 10.1063/1.4855116

Unique chemical reactivity of a graphene nanoribbon's zigzag edge

The Journal of Chemical Physics 126, 134701 (2007); 10.1063/1.2715558

Structure-property predictions for new planar forms of carbon: Layered phases containing $\mathrm{sp}^{2}$ and sp atoms The Journal of Chemical Physics 87, 6687 (1998); 10.1063/1.453405

Breakdown current density of graphene nanoribbons

Applied Physics Letters 94, 243114 (2009); 10.1063/1.3147183

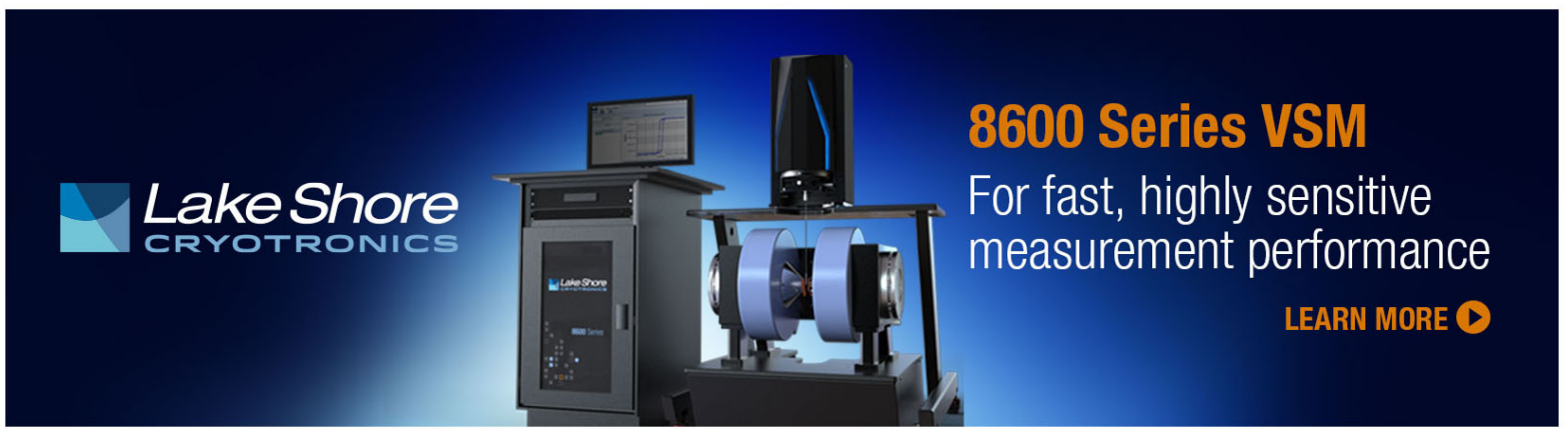




\title{
Spin confinement in the superlattices of graphene ribbons
}

\author{
M. Topsakal, ${ }^{1}$ H. Sevinçli, ${ }^{2}$ and S. Ciraci ${ }^{1,2, a)}$ \\ ${ }^{1}$ UNAM-Institute of Materials Science and Nanotechnology, Bilkent University, 06800 Ankara, Turkey \\ ${ }^{2}$ Department of Physics, Bilkent University, 06800 Ankara, Turkey
}

(Received 4 March 2008; accepted 12 April 2008; published online 1 May 2008)

\begin{abstract}
Based on first-principles calculations, we showed that repeated heterostructures of zigzag graphene nanoribbons of different widths form multiple quantum well structures. Edge states of specific spin directions can be confined in these wells. The electronic and magnetic state of the ribbon can be modulated in real space. In specific geometries, the absence of reflection symmetry causes the magnetic ground state of whole heterostructure to change from antiferromagnetic to ferrimagnetic. These quantum structures of different geometries provide unique features for spintronic applications. (C) 2008 American Institute of Physics. [DOI: 10.1063/1.2919525]
\end{abstract}

Charge carriers of two-dimensional honeycomb crystal of graphene ribbons are expected to behave like massless Dirac fermions. Advances in materials growth and control techniques have made the synthesis of the isolated graphene ${ }^{1}$ and its ribbons ${ }^{2}$ in different orientations possible. Recent studies on the quasi one dimensional graphene ribbons revealed interesting size and geometry dependent electronic and magnetic properties. ${ }^{3}$

In this letter, we showed that periodically repeated junctions of segments of zigzag ribbons with different widths can form stable superlattice structures. The energy band gap and magnetic state of the superlattice are modulated in the real space. Edge states with spin polarization can be confined in alternating quantum wells occurring in different segments of ribbons. Even more remarkable is that the antiferromagnetic (AFM) ground state can be changed to ferrimagnetic (FRM) one in asymmetric junctions.

We have performed first-principles plane wave calculations ${ }^{4}$ within density functional theory ${ }^{5}$ (DFT) using projector augmented-wave potentials. ${ }^{6}$ The exchange correlation potential has been approximated by spin polarized generalized gradient approximation ${ }^{7}$ using PW91 functional. ${ }^{8}$ All structures have been treated within supercell geometry using the periodic boundary conditions. A planewave basis set with kinetic energy cutoff of $500 \mathrm{eV}$ has been used. In the self-consistent potential and total energy calculations, the Brillouin zone is sampled by $(45 \times 1 \times 1)$ special $\mathbf{k}$ points for ribbons. This sampling is scaled according to the size of superlattices. All atomic positions and lattice constants are optimized by using the conjugate gradient method where total energy and atomic forces are minimized. The convergence for energy is chosen as $10^{-5} \mathrm{eV}$ between two steps, and the maximum force allowed on each atom is $0.01 \mathrm{eV} / \AA$.

Zigzag graphene ribbons (ZGNR), i.e., ZGNR $(n)$ with $n$ carbon atoms in its unit cell, are characterized by the states at both edges of ribbon with opposite spin polarization. ${ }^{9}$ These edge states attribute an AFM character. Under applied electric field the ribbon can become half-metallic. ${ }^{10}$ Hydrogen saturated ZGNR $(n)$ is an AFM semiconductor and has a band gap $E_{g}$, which consistently decreases for $n>8$, and eventually diminishes as $n \rightarrow \infty$. In the rest of the paper, all zigzag

\footnotetext{
${ }^{a)}$ Electronic mail: ciraci@fen.bilkent.edu.tr.
}

ribbons are hydrogen terminated unless stated otherwise.

Let us now consider segments of two zigzag ribbons of different widths and different lengths, namely, $\operatorname{ZGNR}\left(n_{1}\right)$ and $\operatorname{ZGNR}\left(n_{2}\right)$, which can make superlattice structures ${ }^{11}$ with atomically perfect and periodically repeating junctions. Normally, the superlattice geometry can be generated by periodically carving small pieces from one or both edges of the nanoribbons. ${ }^{2}$ The typical superlatices we considered and their structure parameters are schematically described in Fig. 1. $\operatorname{ZGNR}\left(n_{1}\right) / \operatorname{ZGNR}\left(n_{2}\right)$ superlattices can be viewed as if a thin slab with periodically modulated width in the $x y$ plane. The electronic potential in this slab is lower $(V<0)$ than outside vacuum $(V=0)$. Normally, states in this thin potential slab propagate along the $x$ axis, but the propagation of specific states in $\operatorname{ZGNR}\left(n_{2}\right)$ is hindered by the potential barrier above and below the narrow segment, ZGNR $\left(n_{1}\right)$. Eventually, these states are confined to the wide segments, and in certain cases also to the narrow segments. Here, the confinement of the states has occurred due to the geometry of the

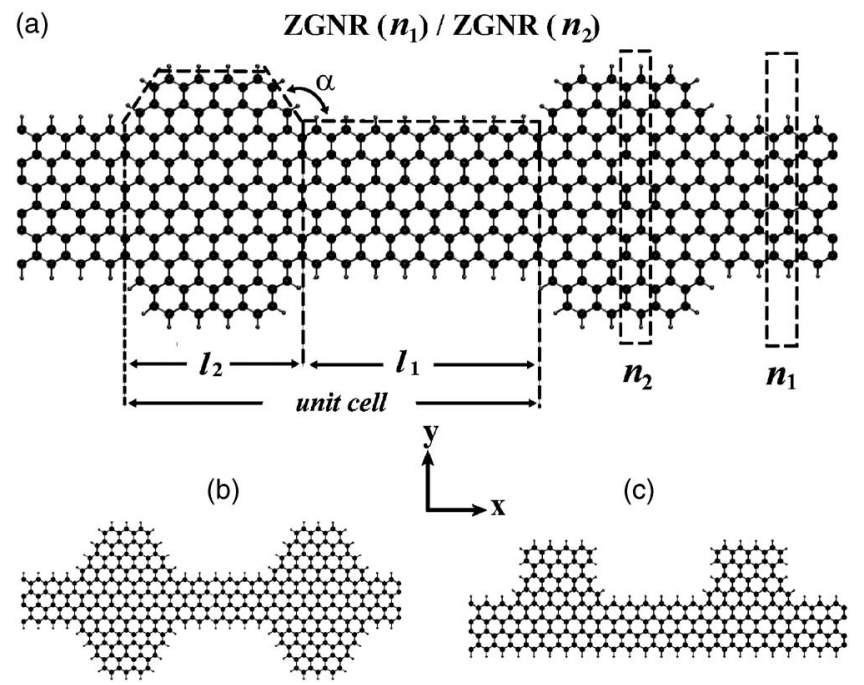

FIG. 1. Typical superlattice structures of ZGNR, ZGNR $\left(n_{1}\right) / \operatorname{ZGNR}\left(n_{2}\right) \cdot n_{1}$ and $n_{2}$ are the number of carbon atoms in the unit cell. $l_{1}$ and $l_{2}$ are lengths of alternating ZGNR segments in numbers of hexagons along the superlattice axis. $\alpha$ is the angle between the $x$ axis and the edge of the intermediate region joining ZGNR $\left(n_{1}\right)$ to $\operatorname{ZGNR}\left(n_{2}\right) . \alpha=120^{\circ}$ for (a) and (b) and $90^{\circ}$ for (c). Dark-large balls and small-light balls indicate carbon and hydrogen atoms, respectively. 


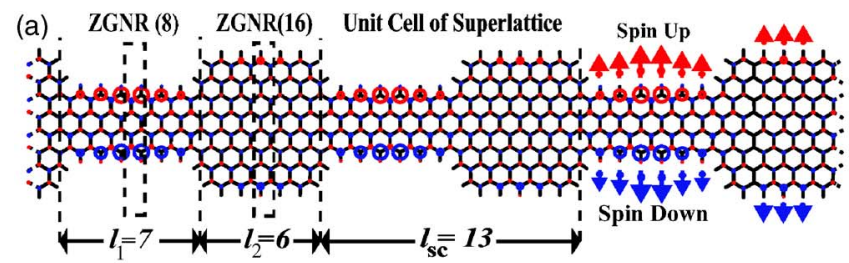

(b)

BANDS

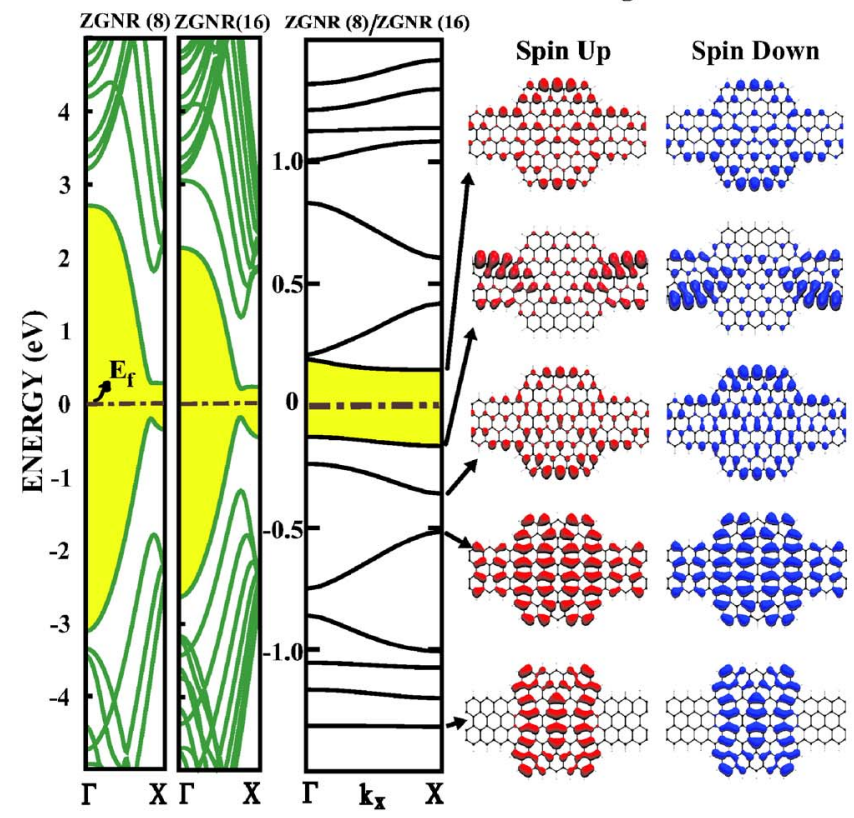

(d)

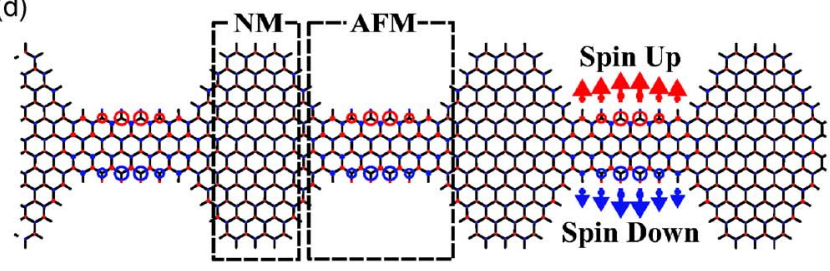

FIG. 2. (Color online) (a) A schematic description of the symmetric ZGNR(8)/ZGNR(16) superlattice with relevant structural parameters. Magnetic moments on the atoms are shown in the left cell by blue (dark) and red (light) circles and arrows for positive and negative values. $l_{\mathrm{sc}}$ is the length of the superlattice unitcells in terms of number of hexagons along the $x$ axis. (b) Energy band structures of AFM ZGNR(8), ZGNR(16) ribbons, and AFM ZGNR(8)/ZGNR(16) superlattice. (c) Charge density isosurfaces of specific superlattice states. Zero of the energy is set to Fermi level, $E_{F}$. The gap between conduction and valence bands are shaded. (d) A specific form of superlattice ZGNR(8)/ZGNR(24) with alternating AFM and NM segments in real space.

system. Defining the confinement in a segment $i$ as $\int_{i}|\Psi(\mathbf{r})|^{2} d \mathbf{r}$, the sharper the interface between $\operatorname{ZGNR}\left(n_{1}\right)$ and $\operatorname{ZGNR}\left(n_{2}\right)$ the stronger becomes the confinement.

In Fig. 2, we show a symmetric superlattice ZGNR(8)/ ZGNR(16). Spin-up and spin-down edge states at the top of the valence band of AFM superlattice are confined to the opposite edges of the narrow segments of the superlattice. Normal flatband states near $-1.2 \mathrm{eV}$ are confined to the wide segments of ZGNR(16). The energy band structure of the superlattice is dramatically different from those of the constituent nanoribbons. If the lengths of the segments are sufficiently large, these segments display the band gap of the corresponding infinite nanoribbon in real space. The total magnetic moment of spin-up and spin-down edge states is zero in each segment, but the magnetic moment due to each edge state is different in adjacent segments. As a result, the
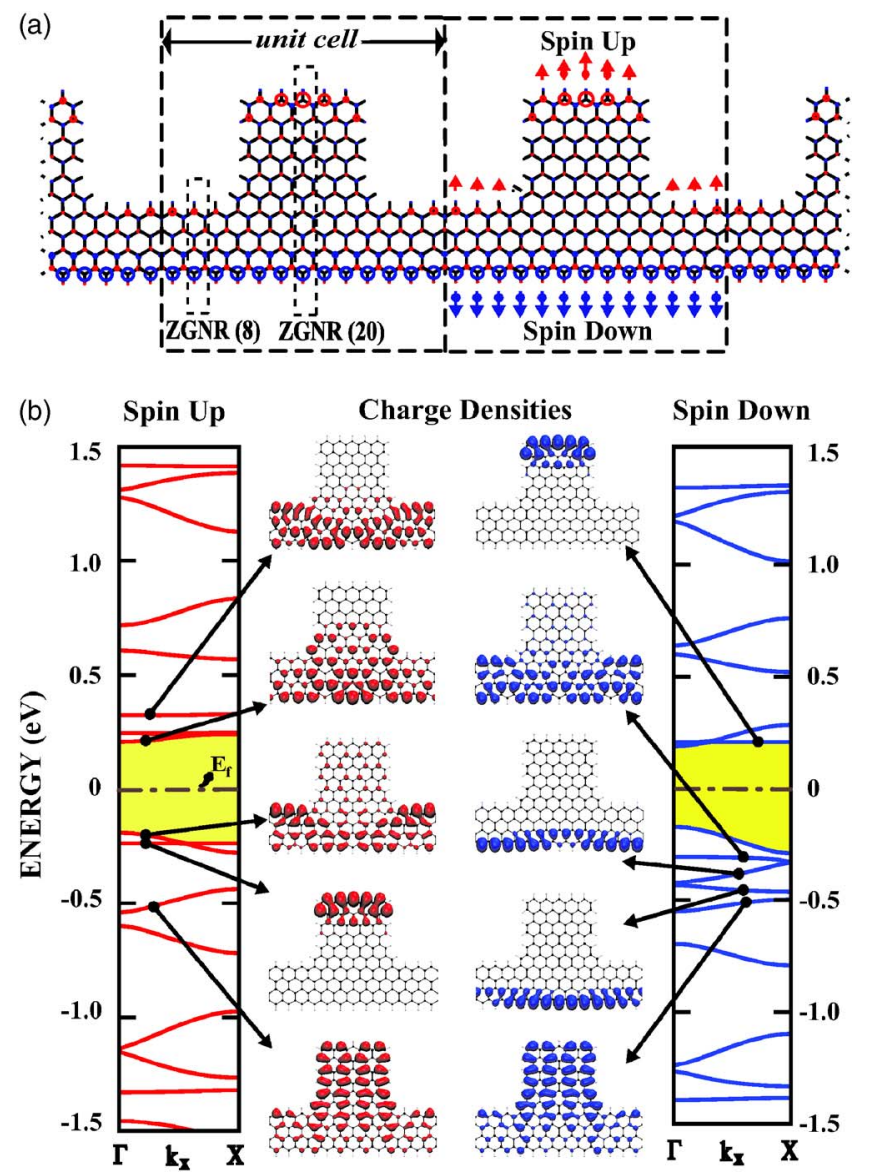

FIG. 3. (Color online) (a) A schematic description of an asymmetric ZGNR(8)/ZGNR(20) superlattice. Total majority and minority spins shown by red (light) and blue (dark) circles (for spin up and spin down, respectively) attribute a FRM behavior. (b) Energy band structure of the FRM semiconductor and charge density isosurfaces of specific propagating and confined states of different spin polarizations.

superlattice is remained to be AFM semiconductor, but the magnitudes of the magnetic moments of the edge states are modulated along the $x$ axis. The coupling between the magnetic moments localized in the neighboring segments is calculated to be $15 \mathrm{meV}$ per unit cell. The modulation of magnetic moments can be controlled by the geometry of the superlattice. For example, as shown in Fig. 2(d), the magnetic moments of the atoms in the wide segment are practically zero and hence the superlattice is composed of AFM and nonmagnetic (NM) segments. However, as $l_{2} \rightarrow 10$ the magnetic moments of the edge atoms at the wide segment become significant.

The situation is even more interesting for an asymmetric superlattice, as shown in Fig. 3. While the spin-down states remain propagating at the flat edge of the superlattice, spin-up states are predominantly confined at the opposite edge of the wide segments. Confinement of states and absence of reflection symmetry breaks the symmetry between spin-up and spin-down edge states. Hence superlattice formation ends up with a FRM semiconductor having different band gaps for different spin states. In agreement with Lieb's theorem, ${ }^{12,13}$ the net magnetic moment calculated to be $2 \mu_{B}$ is equal to the difference of the number of atoms belonging to different sublattices. Flatbands at the edges of spin-up valence band and spin-down conduction band are of particular interest. The spin states of these bands are confined at the 
discontinuous edges of the wide segment which behave as a quantum well. Since a device consisting of a finite size superlatice connected to two electrodes from both ends has high conductance for one spin direction, but low conductance for the opposite one, it operates as a spin valve. Moreover, spin-down electrons injected to this device are trapped in one of the quantum wells generated in a wide segment. As a final remark, we note that the DFT method underestimates the band gaps found in this work. ${ }^{14}$ However, this situation does not affect our conclusions in any essential manner.

In conclusion, through periodic modulation of the width of ZGNR in real space, one can also modulate the electronic structure and magnetic state in real space. Spins can be confined in quantum wells and the AFM ribbon can change to a FRM semiconductor. Since a finite superlattice with desired geometry can now be produced from graphene sheet by chemical methods, ${ }^{2}$ the modulation of electronic and magnetic states, and confined spins hold the promise for the fabrication of interesting nanodevices. For example, by applying a gate voltage through an electrode to a graphene sheet over an oxide layer, one can also generate the desired geometry. The electronic and magnetic properties of superlattices revealed in this study are important for future spintronic applications.

Part of the computations have been performed at the National Center for High Performance Computing of Turkey, Istanbul Technical University (UYBHM).
${ }^{1}$ K. S. Novoselov, A. K. Geim, S. V. Morozov, D. Jiang, Y. Zhang, S. V. Dubonos, I. V. Grigorieva, and A. A. Firsov, Science 306, 666 (2004); K. S. Novoselov, A. K. Geim, S. V. Morozov, D. Jiang, M. I. Katsnelson, I. V. Grigorieva, S. V. Dubonos, and A. A. Firsov, Nature (London) 438, 197 (2005); Y. Zhang, Y.-W. Tan, H. L. Stormer, and P. Kim, ibid. 438, 201 (2005).

${ }^{2}$ X. Li, X. Wang, L. Zhang, S. Lee, and H. Dai, Science 319, 1229 (2008).

${ }^{3}$ V. Barone, O. Hod, and G. E. Scuseria, Nano Lett. 6, 2748 (2006); M. Y. Han, B. Özyilmaz, Y. Zhang, and P. Kim, Phys. Rev. Lett. 98, 206805 (2007); Y.-W. Son, M. L. Cohen, and S. G. Louie, ibid. 97, 216803 (2006); L. Pisani, J. A. Chan, B. Montanari, and N. M. Harrison, Phys. Rev. B 75, 064418 (2007); K. Nakada, M. Fujita, G. Dresselhaus, and M. S. Dresselhaus, ibid. 54, 17954 (1996); Y. Miyamoto, K. Nakada, and M. Fujita, ibid. 59, 9858 (1998); M. Ezawa, ibid. 73, 045432 (2006); O. V. Yazyev and M. I. Katsnelson, Phys. Rev. Lett. 100, 047209 (2008).

${ }^{4}$ G. Kresse and J. Hafner, Phys. Rev. B 47, 558 (1993); G. Kresse and J. Furthmuller, ibid. 54, 11169 (1996).

${ }^{5}$ W. Kohn and L. J. Sham, Phys. Rev. 140, A1133 (1965); P. Hohenberg and W. Kohn, Phys. Rev. B 136, B864 (1964).

${ }^{6}$ P. E. Blöchl, Phys. Rev. B 50, 17953 (1994).

${ }^{7}$ J. P. Perdew, K. Burke, and M. Ernzerhof, Phys. Rev. Lett. 77, 3865 (1996).

${ }^{8}$ J. P. Perdew, J. A. Chevary, S. H. Vosko, K. A. Jackson, M. R. Pederson, D. J. Singh, and C. Fiolhais, Phys. Rev. B 46, 6671 (1992).

${ }^{9}$ M. Fujita, K. Wakabayashi, K. Nakada, and K. Kusakabe, J. Phys. Soc. Jpn. 65, 1920 (1996).

${ }^{10}$ Y.-W. Son, M. L. Cohen, and S. G. Louie, Nature (London) 444, 347 (2006); E. Rudberg, P. Salek, and Y. Luo, Nano Lett. 7, 2211 (2007).

${ }^{11}$ L. Esaki and L. L. Chang, Phys. Rev. Lett. 33, 495 (1974).

${ }^{12}$ E. H. Lieb, Phys. Rev. Lett. 62, 1201 (1989).

${ }^{13}$ J. F.-Rossier and J. J. Palacios, Phys. Rev. Lett. 99, 177204 (2007).

${ }^{14}$ L. Yang, C.-H. Park, Y.-W. Son, M. L. Cohen, and S. G. Louie, Phys. Rev. Lett. 99, 186801 (2007). 